JOURNAL OF THEORETICAL

AND APPLIED MECHANICS

54, 4, pp. 1095-1108, Warsaw 2016

DOI: $10.15632 /$ jtam-pl.54.4.1095

\title{
NONLINEAR VIBRATIONS OF PERIODIC BEAMS
}

\author{
Łukasz Domagalski, JarosŁaW JęDrysiak \\ Lodz University of Technology, Department of Structural Mechanics, Lódź, Poland \\ e-mail: lukasz.domagalski@p.lodz.pl; jarek@p.lodz.pl
}

\begin{abstract}
Geometrically nonlinear vibrations of beams with properties periodically varying along the axis are investigated. The tolerance method of averaging differential operators with highly oscillating coefficients is applied to obtain governing equations with constant coefficients. The proposed model describes dynamics of the beam with the effect of microstructure size. In an example, an analysis of undamped forced nonlinear vibrations of the periodic beam is shown. Moreover, the results obtained for undamped free vibrations of periodic beams by the tolerance model are justified by those results from the finite element method. These results can be used as a benchmark in similar problems.
\end{abstract}

Keywords: nonlinear vibrations, periodic beams, tolerance modelling

\section{Introduction}

The paper concerns with geometrically nonlinear vibrations of beams with geometric and material properties periodically varying along the $x$-axis. Moreover, such beams can interact with a periodically inhomogeneous viscoelastic subsoil. A fragment of such a beam is shown in Fig. 1.

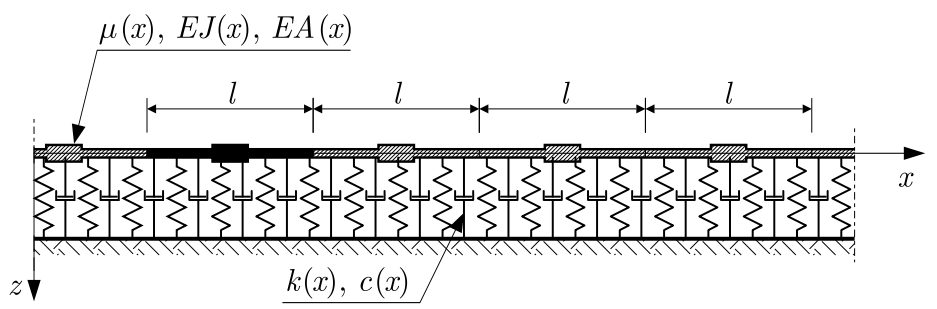

Fig. 1. A fragment of a periodic beam

Equations of motion of such structures have usually highly oscillating, periodic, non-continuous functional coefficients. For this reason, emphasis is placed on the formulation of continuous models of the considered structures. In the proposed method of modelling, this is performed by substituting the original equations with an effective model with constant coefficients. This makes it possible to avoid full discretization of the problem.

Structures with physical properties regularly arranged in the body domain are commonly found in nature and are widely used in engineering. The continuous interest in such objects is due to their specific properties. Properly designed composite structures are characterized by, among others, favourable ratio of stiffness to weight. This, due to the trends in modern technology for the design of lightweight, high-strength structures, indicates actuality of the problem. In particular, periodic structures exhibit some interesting and desirable dynamic properties, namely, they may serve as filters for some specific vibration frequency bands, cf. Banakh and Kempner (2010). Analysis of the so-called locally resonant beams were published in numerous papers, e.g. by Olhoff et al. (2012), where the optimization of beam geometry in order to obtain the maximum width of the frequency band-gap leads to a periodic structure. 
Direct numerical modelling of structures of this kind, such as finite element discretization, is one of the possible ways of analysis the considered problems. The computational cost of the parametric analysis within the discretization approach is, however, proportional to the variation range of the parameters under consideration. Thus, it is advisable to strive to formulate alternative continuous models in order to reduce the computational cost.

Among the analytical methods applied in stationary problems of periodic structures, the most widespread are those based on the rigorous mathematical theory of asymptotic homogenization of differential operators. In this approach, the actual periodic structure is modelled as a homogeneous anisotropic structure with some effective properties. The above-mentioned effective properties are obtained through analysis of the so-called periodicity cell problem. The fundamentals of this theory are described by Bensoussan et al. (1978), Sanchez-Palencia (1980), Bakhvalov and Panasenko (1984), Jikov et al. (1994), Lewiński and Telega (2000), Krysko et al. (2008). Some various works are devoted to derivation of micro-periodic beam equilibrium equations in the frame of homogenization theory, wherein the starting point of analysis are three-dimensional elasticity theory equations, see Kolpakov (1991, 1995, 1998, 1999), Syerko et al. (2013). Certain analytical approaches and the finite element method are also used to evaluate strength and buckling of sandwich beams having corrugated cores, e.g. by Magnucki et al. (2013).

The literature on the problems of linear vibrations of periodic beams is extensive. In most of the research papers, attention is focused on local resonance properties of such structures. The two-scale asymptotic expansions are applied by He et al. (2013) in analysis of beams with periodically variable stiffness. A common approach making use of the theory of Floquet-Bloch waves in the analysis. This was applied in the analysis of the Timoshenko (Chen and Wang, 2013) and Euler-Bernoulli (Chen, 2013) beam vibrations. The problem of wave propagation in a periodic elastically supported beam was considered by Yu et al. (2012) using the transfer matrix method. The direct approach with use of the Heaviside step function in the case of the forced oscillation of the plate band on a periodic elastic substrate was applied by Sylvia and Hull (2013), where the investigations were brought to a one-dimensional problem.

Description of dynamic problems within geometrically linear theories imposes severe restrictions on deformations, limiting the displacements order to the smallest dimension of the structural element considered. Since the considered structures are slender at the macro level, this limitation makes it impossible to correctly analyze the whole spectrum of their applicability. In addition, some physical phenomena that occur in vibrations of nonlinear systems which have a significant impact on motion characteristics are impossible to investigate in terms of linearized theories. Nonlinear vibrations of homogeneous nano-beams cooperating with a homogeneous visco-elastic substrate were considered by Wang and $\mathrm{Li}$ (2014), vibrations of the sandwich beams by Krysko et al. (2008). The paper by Awrejcewicz et al. (2011) contains comparison of nonlinear vibration models of the Euler-Bernoulli beam derived through FEM discretization and the finite differences method. Despite the large number of studies dealing with non-linear vibrations (e.g. Sedighi et al., 2013; Hryniewicz and Kozioł, 2013), the majority of publications relates to systems with a relatively small number of degrees of freedom.

In this contribution, in order to replace differential equations with highly oscillating coefficients by equations with constant coefficients, the tolerance modelling, see Woźniak and Wierzbicki (2000), Woźniak et al. $(2008,2010)$ is applied. This approach was introduced for the purpose of analysis of various thermomechanical problems of periodic elastic composites in a series of papers, e.g. for thin periodic plates on a foundation by Jędrysiak (1999), for micro-periodic beams under moving load by Mazur-Śniady and Śniady (2001),for periodic beams for plates with the microstructure size of an order of the plate thickness - for periodic thin by Mazur-Śniady et al. (2004), for periodic medium-thickness by Baron (2006), for thin functionally graded by Jędrysiak (2013), for multiperiodic fibre reinforced composites by Jędrysiak and 
Woźniak (2006), for periodic shells by Tomczyk (2007), for functionally graded plates by Wirowski (2012). This technique was also used in vibration analysis of periodic beams within the linear theory by Mazur-Śniady (1993), where the equations of motion and their generalization by including the influence of the axial force, elastic subsoil and viscous damping were derived. The books by Woźniak and Wierzbicki (2000), Woźniak et al. (2008, 2010) contain the fundamentals of this theory and numerous examples of application.

The main aim of this note is to derive averaged governing equations of the nonlinear tolerance model of dynamics of periodic beams on a viscoelastic foundation and show a certain application of this model to a special problem. Moreover, some justifications of the results by the proposed model are presented by the results obtained from the finite element method for a benchmark problem of free vibrations of a linear periodic beam.

\section{Formulation of the problem}

The object under consideration is a linearly elastic prismatic beam, bilaterally interacting with a periodic viscoelastic foundation. Let $O x y z$ be an orthogonal Cartesian coordinate system in which the $O x$ axis coincides with the axis of the beam, the cross section of the beam is symmetric with respect to the plane of the load $O x z$, the load acts in the direction of the axis $O z$. The problem can be treated as one-dimensional, so that we define the region occupied by the beam as $\Omega \equiv[0, L]$, where $L$ stands for the beam length.

The beam is assumed to be made of many repetitive small elements, called periodicity cells, defined as $\Delta \equiv[-l / 2, l / 2]$, where $l \ll L$ is length of the cell and named the microstructure parameter.

Our considerations are based on the Rayleigh theory of beams with von Kármán type nonlinearity. Since we are interested in the transverse vibrations only, the effect of axial inertia is neglected in further considerations. Let $\partial^{k}=\partial^{k} / \partial x^{k}$ be the $k$-th derivative of a function with respect to the $x$ coordinate, overdot stands for the derivative with respect to time. Let $w=w(x, t)$ be the transverse deflection, $u_{0}=u_{0}(x, t)$ longitudinal displacement, $E A=E(x) A(x)$ and $E(x) J=E J(x)$ tensile and flexural stiffness, $k=k(x)$ and $c=c(x)$ - elasticity and damping coefficients of the foundation, $\mu=\mu(x)$ and $\vartheta=\vartheta(x)$ mass and rotational moment of inertia per unit length and $q=q(x, t)$ - transverse load. The strain and kinetic energy density per unit length of the beam are

$$
\mathcal{W}=\frac{1}{2} E A\left(\partial u_{0}+\frac{1}{2} \partial w \partial w\right)^{2}+\frac{1}{2} E J\left(\partial^{2} w\right)^{2} \quad \mathcal{K}=\frac{1}{2} \mu \dot{w} \dot{w}+\frac{1}{2} \vartheta \partial \dot{w} \partial \dot{w}
$$

For the subsoil, we apply the Kelvin-Voight model, so that the dissipative force is assumed in the form

$$
p=p(x, t)=c(x) \dot{w}(x, t)
$$

The equations of motion can be obtain from the extended (Woźniak et al., 2010) principle of stationary action $\mathcal{A}=\mathcal{A}\left(u_{0}, w\right)$ formulated as

$$
\begin{aligned}
\delta \mathcal{A} & =\delta \int_{0}^{1} \int_{0}^{L} \mathcal{L} d x d t=\int_{0}^{1} \int_{0}^{L} \delta \mathcal{L} d x d t=\int_{0}^{1} \int_{0}^{L}\left[\left(\frac{\partial \mathcal{L}}{\partial u_{0}}-\partial \frac{\partial \mathcal{L}}{\partial\left(\partial u_{0}\right)}\right) \delta u_{0}\right. \\
& \left.+\left(\frac{\partial \mathcal{L}}{\partial w}-\partial \frac{\partial \mathcal{L}}{\partial(\partial w)}+\partial^{2} \frac{\partial \mathcal{L}}{\partial\left(\partial^{2} w\right)}-\frac{d}{d t} \frac{\partial \mathcal{L}}{\partial \dot{w}}+\frac{d}{d t} \partial \frac{\partial \mathcal{L}}{\partial(\partial \dot{w})}\right) \delta w\right] d x d t=0
\end{aligned}
$$


where the Lagrangian is

$$
\begin{aligned}
& \mathcal{L}\left(x, t, w, \dot{w}, \partial w, \partial^{2} w, \partial u_{0}\right)=\mathcal{W}-\mathcal{K}+p w+\frac{1}{2} k w w-q w \\
& \quad=\frac{1}{2} E A\left(\partial u_{0}+\frac{1}{2} \partial w \partial w\right)^{2}+\frac{1}{2} \partial^{2} w E J \partial^{2} w-\frac{1}{2} \mu \dot{w} \dot{w}-\frac{1}{2} \vartheta \partial \dot{w} \partial \dot{w}+p w+\frac{1}{2} k w w-q w
\end{aligned}
$$

The system of nonlinear coupled differential equations for the longitudinal displacements $u_{0}$ and the transverse deflection $w$ resulting from (2.3) can be written as

$$
\begin{aligned}
& \partial\left[E A\left(\partial u_{0}+\frac{1}{2} \partial w \partial w\right)\right]=0 \\
& \mu \ddot{w}-\partial(\vartheta \partial \ddot{w})+c \dot{w}+k w+\partial^{2}\left(E J \partial^{2} w\right)-\partial\left[E A\left(\partial u_{0}+\frac{1}{2} \partial w \partial w\right) \partial w\right]=q
\end{aligned}
$$

The coefficients $E A, E J, k, \mu, \vartheta, c$ are highly oscillating, often non-continuous functions of the $x$-coordinate. The main aim of this note is to derive an approximately equivalent model, which describes geometrically nonlinear vibrations of periodic beams bilaterally interacting with a periodic viscoelastic foundation, taking into account the effect of microstructure size.

\section{Introductory concepts and basic assumptions of the tolerance modelling}

The averaged equations of periodic beams with large deflections are derived using the concepts and assumptions of the tolerance modelling technique, see Woźniak et al. (2010). The fundamental concepts are: the tolerance system, averaging operation and certain classes of functions such as the tolerance-periodic $(T P)$, slowly-varying $(S V)$, highly oscillating $(H O)$ and fluctuation shape $(F S)$ functions. The tolerance parameter, associated with the tolerance relation, is denoted by $d, 0<d \ll 1$. The highest order of function derivative that can be included into a certain function class is denoted by $\alpha$.

Let $\Delta(x)=x+\Delta, \Omega_{\Delta}=\{x \in \Omega: \Delta(x) \subset \Omega\}$ be a cell with its center at $x \in \Omega_{\Delta}$. The averaging operator for an arbitrary integrable function $f$ is defined by

$$
\langle f\rangle(x)=l^{-1} \int_{\Delta(x)} f(y) d y \quad x \in \Omega_{\Delta} \quad y \in \Delta(x)
$$

It can be shown (Woźniak et al., 2010) that for a periodic function $f$ of $x$, its average is constant.

The first of the basic assumptions is the micro-macro decomposition of the unknown transverse deflection and longitudinal displacement

$$
\begin{aligned}
& w(x, t)=W(x, t)+h^{A}(x) V^{A}(x, t) \\
& A=1, \ldots, N \quad W(\cdot, t), V^{A}(\cdot, t) \in S V_{d}^{2}(\Omega, \Delta) \quad h^{A}(\cdot) \in F S_{d}^{2}(\Omega, \Delta)
\end{aligned}
$$

and

$$
\begin{aligned}
& u_{0}(x, t)=U(x, t)+g^{K}(x) T^{K}(x, t) \\
& K=1, \ldots, M \quad U(\cdot, t), T^{K}(\cdot, t) \in S V_{d}^{1}(\Omega, \Delta) \quad g^{K}(\cdot) \in F S_{d}^{1}(\Omega, \Delta)
\end{aligned}
$$

Here and hereafter, the summation convention holds.

The new basic kinematic unknowns $W(\cdot)$ and $U(\cdot)$ are called the transverse and the axial macrodisplacements, respectively; $V^{A}(\cdot)$ and $T^{K}(\cdot)$ are additional kinematic unknowns, called the fluctuation amplitudes. The unknown functions are assumed to be slowly-varying. The highly oscillating fluctuation shape functions $h^{A}$ and $g^{K}$ are postulated a priori in every problem 
under consideration and are assumed to describe unknown fields oscillations caused by structure inhomogeneity. These functions have to satisfy the following conditions

$$
\begin{aligned}
& \left\langle\mu h^{A}\right\rangle=0 \quad\left\langle\mu g^{K}\right\rangle=0\left\langle\mu h^{A} h^{B}\right\rangle=0 \quad \text { for } \quad A \neq B \\
& \left\langle\mu g^{K} g^{L}\right\rangle=0 \quad \text { for } \quad K \neq L \quad \partial^{m} h^{A} \in O\left(l^{2-m}\right) \\
& \partial^{n} g^{K} \in O\left(l^{1-m}\right) \quad A, B=1, \ldots, N \quad K, L=1, \ldots, M
\end{aligned}
$$

The second assumption is the tolerance averaging approximation

$$
\begin{aligned}
& \langle f\rangle(x)=\left\langle f_{x}\right\rangle(x)+O(d) \quad\left\langle f \partial^{\alpha}\left(\phi^{A} \Psi\right)\right\rangle(x)=\left\langle f \partial^{\alpha} \phi^{A}\right\rangle(x) \Psi(x)+O(d) \\
& \langle f \Psi\rangle(x)=\langle f\rangle(x) \Psi(x)+O(d) \quad\left\langle f \partial\left(\gamma^{K} \Xi\right)\right\rangle(x)=\left\langle f \partial \gamma^{K}\right\rangle(x) \Xi(x)+O(d) \\
& \langle f \Xi\rangle(x)=\langle f\rangle(x) \Xi(x)+O(d) \quad f \in T P_{d}^{\alpha}(\Omega, \Delta) \quad \Psi \in S V_{d}^{2}(\Omega, \Delta) \\
& \phi^{A} \in F S_{d}^{2}(\Omega, \Delta) \quad \Xi \in S V_{d}^{1}(\Omega, \Delta) \quad \gamma^{K} \in F S_{d}^{1}(\Omega, \Delta) \\
& x \in \Omega \quad \alpha=1,2 \quad A=1, \ldots, N \quad K=1, \ldots, M \quad 0<d \ll 1
\end{aligned}
$$

in which the terms of the order of the tolerance parameter $O(d)$ are assumed to be negligibly small.

\section{The governing equations of the proposed models}

\subsection{The equations of the tolerance model}

After substitution of micro-macro decompositions (3.2) and (3.3) into Lagrangian (2.4), the next step of modelling is averaging (3.1) over an arbitrary periodicity cell with approximations (3.5).

The averaged action functional has the following form

$$
\delta \mathcal{A}_{h}=\delta \int_{0}^{1} \int_{0}^{L}\left\langle\mathcal{L}_{h}\right\rangle d x d t=\int_{0}^{1} \int_{0}^{L} \delta\left\langle\mathcal{L}_{h}\right\rangle d x d t=0
$$

where the averaged Lagrangian is formulated as follows

$$
\begin{aligned}
\left\langle\mathcal{L}_{h}\right\rangle & =\frac{1}{2} D \partial^{2} W \partial^{2} W+D^{A} \partial^{2} W V^{A}+(P-Q) W+\frac{1}{2} K W W+\frac{1}{2} B\left(\partial U+\frac{1}{2} \partial W \partial W\right)^{2} \\
& +l^{2}\left(P^{A}-Q^{A}+K^{A} W+\frac{1}{2} l^{2} K^{A B} V^{B}\right) V^{A}-\frac{1}{2} M \dot{W} \dot{W}-\frac{1}{2} J \partial \dot{W} \partial \dot{W} \\
& -\left(l^{2} M^{A} \dot{W}+l J^{A} \partial \dot{W}\right) \dot{V}^{A}+\left[B^{K}\left(\partial U+\frac{1}{2} \partial W \partial W\right)+\frac{1}{2} B^{K L} T^{L}\right] T^{K} \\
& -\frac{1}{2} l^{2}\left(l^{2} M^{A B}+J^{A B}\right) \dot{V}^{B} \dot{V}^{A}+l\left[B^{A}\left(\partial U+\frac{1}{2} \partial W \partial W\right)+B^{A K} T^{K}\right] V^{A} \partial W \\
& +\frac{1}{2} l^{2}\left[B^{A B}\left(\partial U+\frac{3}{2} \partial W \partial W\right)+B^{A B K} T^{K}\right] V^{B} V^{A} \\
& +\frac{1}{2} l^{3}\left(B^{A B C} \partial W+\frac{1}{4} l B^{A B C D} V^{D}\right) V^{A} V^{B} V^{C}+\frac{1}{2} D^{A B} V^{A} V^{B}
\end{aligned}
$$

Under essential boundary conditions it leads to a system of Euler-Lagrange equations

$$
\begin{aligned}
& \frac{\partial\left\langle\mathcal{L}_{h}\right\rangle}{\partial U}-\partial \frac{\partial\left\langle\mathcal{L}_{h}\right\rangle}{\partial(\partial U)}=0 \\
& \frac{\partial\left\langle\mathcal{L}_{h}\right\rangle}{\partial W}-\partial \frac{\partial\left\langle\mathcal{L}_{h}\right\rangle}{\partial(\partial W)}+\partial^{2} \frac{\partial\left\langle\mathcal{L}_{h}\right\rangle}{\partial\left(\partial^{2} W\right)}-\frac{d}{d t} \frac{\partial\left\langle\mathcal{L}_{h}\right\rangle}{\partial(\dot{W})}+\frac{d}{d t}\left(\partial \frac{\partial\left\langle\mathcal{L}_{h}\right\rangle}{\partial(\partial \dot{W})}\right)=0 \\
& \frac{\partial\left\langle\mathcal{L}_{h}\right\rangle}{\partial T^{K}}=0 \quad \frac{\partial\left\langle\mathcal{L}_{h}\right\rangle}{\partial V^{A}}-\frac{d}{d t} \frac{\partial\left\langle\mathcal{L}_{h}\right\rangle}{\partial\left(\dot{W}^{A}\right)}=0
\end{aligned}
$$


After some manipulations, we arrive at the following system of equations

$$
\begin{aligned}
& \partial\left(B \partial U+B^{K} T^{K}\right)+\partial\left(\frac{1}{2} B \partial W \partial W+l B^{A} V^{A} \partial W+\frac{1}{2} l^{2} B^{A B} V^{B} V^{A}\right)=0 \\
& D \partial^{4} W+D^{A} \partial^{2} V^{A}+K W+l^{2} K^{A} V^{A}+C \dot{W}+M \ddot{W}-J \partial^{2} \ddot{W} \\
& \quad+l^{2} C^{A} \dot{W}^{A}+l^{2} M^{A} \ddot{V}^{A}-l J^{A} \partial \ddot{V}^{A}-Q \\
& \quad-\partial\left[\left(B\left(\partial U+\frac{1}{2} \partial W \partial W\right)+B^{K} T^{K}+l B^{A} V^{A} \partial W+\frac{1}{2} l^{2} B^{A B} V^{B} V^{A}\right) \partial W\right] \\
& \quad-\partial\left[l B^{A K} V^{A} T^{K}+\left(\partial U+\frac{1}{2} \partial W \partial W\right) l B^{A} V^{A}+l^{2} B^{A B} \partial W V^{B} V^{A}\right. \\
& \left.\quad+\frac{1}{2} l^{3} B^{A B C} V^{A} V^{B} V^{C}\right]=0 \\
& \left(D^{A B}+l^{4} K^{A B}\right) V^{B}+l^{4} C^{A B} \dot{W}^{B}+l^{2}\left(l^{2} M^{A B}+J^{A B}\right) \ddot{V}^{B}+D^{A} \partial^{2} W+l^{2} K^{A} W \\
& \quad+l\left(l M^{A} \ddot{W}+J^{A} \partial \ddot{W}\right)+l^{2} C^{A} \dot{W}-l^{2} Q^{A}+l\left[B^{A}\left(\partial U+\frac{1}{2} \partial W \partial W\right)+B^{A K} T^{K}\right] \partial W \\
& \quad+l^{2}\left(B^{A B} \partial U+\frac{3}{2} B^{A B} \partial W \partial W+B^{A B K} T^{K}+\frac{3}{2} l B^{A B C} V^{C} \partial W\right. \\
& \left.\quad+\frac{1}{2} l^{2} B^{A B C D} V^{C} V^{D}\right) V^{B}=0 \\
& B^{K L} T^{L}+B^{K} \partial U+\frac{1}{2} B^{K} \partial W \partial W+l B^{A K} V^{A} \partial W+\frac{1}{2} l^{2} B^{A B K} V^{B} V^{A}=0
\end{aligned}
$$

with constant coefficients related to the beam properties

$$
\begin{array}{lll}
\langle E A\rangle \equiv B & \left\langle E A \partial h^{A}\right\rangle \equiv l B^{A} & \langle\mu\rangle \equiv M \\
\left\langle E A \partial g^{K}\right\rangle \equiv B^{K} & \left\langle E A \partial h^{A} \partial g^{K}\right\rangle \equiv l B^{A K} & \left\langle\mu h^{A}\right\rangle \equiv l^{2} M^{A} \\
\left\langle E A \partial g^{K} \partial g^{L}\right\rangle \equiv B^{K L} & \left\langle E A \partial h^{A} \partial h^{B} \partial g^{K}\right\rangle \equiv l^{2} B^{A B K} & \left\langle\mu h^{A} h^{B}\right\rangle \equiv l^{4} M^{A B} \\
\langle E J\rangle \equiv D & \left\langle E A \partial h^{A} \partial h^{B}\right\rangle \equiv l^{2} B^{A B} & \langle\vartheta\rangle \equiv J \\
\left\langle E J \partial^{2} h^{A}\right\rangle \equiv D^{A} & \left\langle E A \partial h^{A} \partial h^{B} \partial h^{C}\right\rangle \equiv l^{3} B^{A B C} & \left\langle\vartheta \partial h^{A}\right\rangle \equiv l J^{A} \\
\left\langle E J \partial^{2} h^{B} \partial^{2} h^{A}\right\rangle \equiv D^{A B} & \left\langle E A \partial h^{A} \partial h^{B} \partial h^{C} \partial h^{D}\right\rangle \equiv l^{4} B^{A B C D} & \left\langle\vartheta \partial h^{A} \partial h^{B}\right\rangle \equiv l^{2} J^{A B}
\end{array}
$$

to the subsoil properties and to the transverse load

$$
\begin{array}{lll}
\langle c\rangle \equiv C & \langle k\rangle \equiv K & \langle q\rangle \equiv Q \\
\left\langle c h^{A}\right\rangle \equiv l^{2} C^{A} & \left\langle k h^{A}\right\rangle \equiv l^{2} K^{A} & \left\langle q h^{A}\right\rangle \equiv l^{2} Q^{A} \\
\left\langle c h^{A} h^{B}\right\rangle \equiv l^{4} C^{A B} & \left\langle k h^{A} h^{B}\right\rangle \equiv l^{4} K^{A B} &
\end{array}
$$

It is a system of $2+N+M$ differential equations for the macrodisplacements $U(\cdot), W(\cdot)$ and for the fluctuation amplitudes of the axial displacement $T^{K}(\cdot)$ and of the deflection $V^{A}(\cdot)$. The coefficients of these equations are constant, some of them depend on the size $l$ of the periodicity cell.

Equations(4.4) can be simplified to the following form

$$
\begin{aligned}
& D \partial^{4} W+D^{A} \partial^{2} V^{A}+K W+l^{2} K^{A} V^{A}-\bar{N} \partial^{2} W-l \widetilde{N}^{A} \partial V^{A} \\
& \quad+C \dot{W}+M \ddot{W}-J \partial^{2} \ddot{W}+l^{2} C^{A} \dot{W}^{A}+l^{2} M^{A} \ddot{V}^{A}-l J^{A} \partial \ddot{V}^{A}-Q=0 \\
& \quad\left(D^{A B}+l^{4} K^{A B}\right) V^{B}+l^{4} C^{A B} \dot{W}^{B}+l^{2}\left(l^{2} M^{A B}+J^{A B}\right) \ddot{V}^{B}+l \widetilde{N}^{A} \partial W+l^{2} \widetilde{N}^{A B} V^{B} \\
& \quad+D^{A} \partial^{2} W+l^{2} K^{A} W+l\left(l M^{A} \ddot{W}+J^{A} \partial \ddot{W}\right)+l^{2} C^{A} \dot{W}-l^{2} Q^{A}=0
\end{aligned}
$$

The nonlinear terms (underlined) involve the axial force

$$
N=E A \varepsilon_{0}=E A\left(\partial u_{0}+\frac{1}{2} \partial w \partial w\right)
$$


averaged with the certain gradients of the fluctuation shape function as weights

$$
\begin{aligned}
& \left\{\begin{array}{c}
\bar{N} \\
l \widetilde{N}^{A} \\
l^{2} \widetilde{N}^{A B}
\end{array}\right\}=\left\{\begin{array}{c}
\langle N\rangle \\
\left\langle N \partial h^{A}\right\rangle \\
\left\langle N \partial h^{A} \partial h^{B}\right\rangle
\end{array}\right\} \\
& =\left[\begin{array}{ccc}
\bar{B}_{0} & l \bar{B}_{0}^{C} & l^{2} \bar{B}_{0}^{C D} \\
l \bar{B}_{0}^{A} & l^{2} \widetilde{B}_{0}^{A C} & l^{3} \bar{B}_{0}^{A C D} \\
l^{2} \bar{B}_{0}^{A B} & l^{3} \bar{B}_{0}^{A B C} & l^{4} \bar{B}_{0}^{A B C D}
\end{array}\right]\left(\frac{1}{L} \int_{0}^{L}\left\{\begin{array}{c}
\frac{1}{2} \partial W \partial W \\
V^{C} \partial W \\
\frac{1}{2} V^{C} V^{D}
\end{array}\right\} d x+\left\{\begin{array}{c}
\delta_{0} \\
0 \\
0
\end{array}\right\}\right)
\end{aligned}
$$

where

$$
\delta_{0}=L^{-1} \int_{0}^{L} \partial U d x=L^{-1}[U(L)-U(0)]
$$

stands for the relative elongation of the beam axis. The coefficients of (4.9) are

$$
\begin{array}{ll}
\bar{B}_{0}=B-B^{L}\left(B^{-1}\right)^{L K} B^{K} & \widetilde{B}_{0}^{A B}=B^{A B}-B^{A K}\left(B^{-1}\right)^{L K} B^{L B} \\
\bar{B}_{0}^{A}=B^{A}-B^{A L}\left(B^{-1}\right)^{L K} B^{K} & \bar{B}_{0}^{A B C}=B^{A B C}-B^{A B L}\left(B^{-1}\right)^{L K} B^{K C} \\
\bar{B}_{0}^{A B}=B^{A B}-B^{A B L}\left(B^{-1}\right)^{L K} B^{K} & \bar{B}_{0}^{A B C D}=B^{A B C D}-B^{A B K}\left(B^{-1}\right)^{K L} B^{L C D}
\end{array}
$$

It can be seen that the axial displacement can be eliminated in a way common in the conventional Euler-Bernoulli or Timoshenko theories of uniform beams.

\subsection{The equations of the tolerance-asymptotic model}

Neglecting in equations (4.4) or (4.7) the terms with the microstructure parameter $l$ and introducing the effective bending stiffness of the beam

$$
D_{0} \equiv D-D^{A}\left(D^{-1}\right)^{A B} D^{B}
$$

we arrive at the equations

$$
\begin{aligned}
& \bar{B}_{0} \partial\left(\partial U+\frac{1}{2} \partial W \partial W\right)=0 \\
& M \ddot{W}-J \partial^{2} \ddot{W}+C \dot{W}+K W+\bar{D}_{0} \partial^{4} W-\bar{B}_{0}\left(\partial U+\frac{1}{2} \partial W \partial W\right) \partial^{2} W=Q
\end{aligned}
$$

The above equations do not describe the effect of the cell size on the behaviour of periodic beams under consideration. Hence, the asymptotic model makes it possible to analyse vibrations on the macrolevel only. In the framework of this approximation, certain higher eigenfrequencies and eigenforms of vibrations cannot be obtained.

\section{Examples of application}

Let us consider a hinged-hinged beam with immovable ends. The beam has a constant cross section and is provided by a system of periodically distributed system of concentrated masses $M_{1}, M_{2}$ with rotational inertia $I_{1}, I_{2}$, as it is shown in Fig. 2.

A single cell is shown in Fig. 3. The mass distribution in a periodicity cell is given by

$$
\begin{aligned}
& \mu(y)=\mu_{0}+M_{1} \delta(y)+M_{2} \delta\left(y+\frac{l}{2}\right) \\
& \vartheta(y)=\vartheta_{0}+I_{1} \delta(y)+I_{2} \delta\left(y+\frac{l}{2}\right) \quad y \in(x)
\end{aligned}
$$




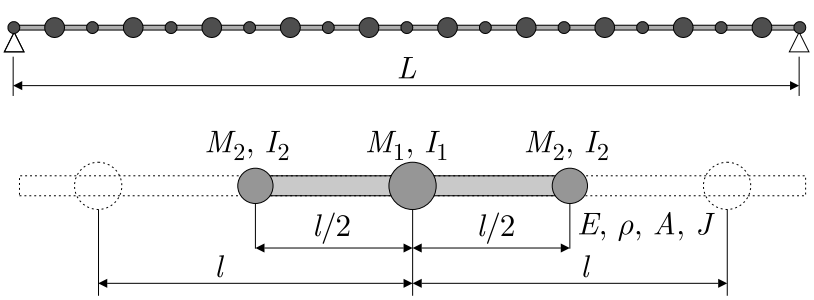

Fig. 2. The considered beam and its fragment

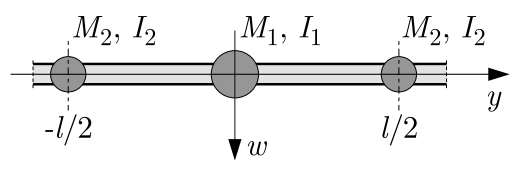

Fig. 3. A single periodicity cell

In this Section, the free undamped and forced vibrations will be analyzed. In the case of forced vibrations, we assume that the transverse load is given by

$$
q(x, t)=q_{0} \sin \left(\frac{\pi x}{L}\right) \cos (\Omega t)
$$

\section{The method of solution}

\subsection{Fluctuation shape functions}

The fundamental assumption of the tolerance approach is the macro-micro decomposition (3.2), (3.3) of the unknown displacements. It can be seen that the fluctuation shape functions (FSF) play a crucial role in the analysis. As it has been mentioned, these functions represent the oscillations of displacements in a periodicity cell. The common practise is to use approximate functions, usually the sine and cosine that are infinitely differentiable. Another way is to utilize the periodic eigenproblem solutions of a periodicity cell, which can be obtained through numerical analysis.

Here, this is done through both the above mentioned ways. For the symmetric periodicity cell (Fig. 3), two transverse and one longitudinal approximate modes of cell vibrations are considered

$$
\begin{array}{ll}
h^{1}(y)=l^{2}\left[\cos \left(\frac{2 A \pi y}{l}\right)+c\right] & h^{2}(y)=l^{2} \sin \left(\frac{2 A \pi y}{l}\right) \\
g^{1}(y)=l\left[\cos \left(\frac{2 K \pi y}{l}\right)+c\right] & c=\frac{M_{2}-M_{1}}{\mu_{0} l+M_{1}+M_{2}}
\end{array}
$$

The constant $c$ is calculated from condition $(3.4)_{1}$.

(a)

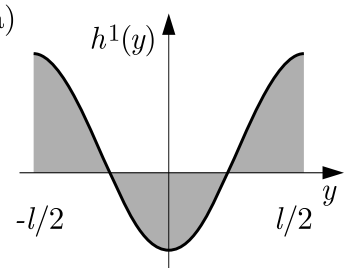

(b)

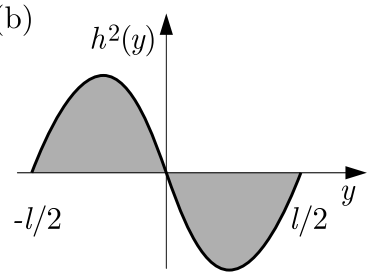

(c)

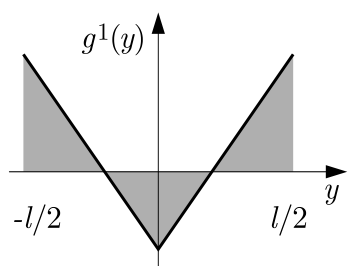

Fig. 4. FE-based fluctuation shape functions: transverse (a), (b), and longitudinal (c) modes of the periodicity cell vibrations

The refined fluctuation shape functions (Fig. 4) are obtained from a finite element analysis of the cell. The calculations are performed in the environment of Maple. The periodicity cell is divided into two elements, and the periodic boundary conditions are assumed. 
Since the fluctuation shape functions satisfy the orthogonality conditions, there is possibility to obtain cell vibrations mode shapes taking more approximate FSFs and observing the convergence of total oscillation on a periodicity cell. In the case of the finite element based cell solution, a similar strategy can be adopted.

\subsection{Solutions of the tolerance model}

The solutions of the tolerance model equations, as well as the loads, are assumed in the form of truncated Fourier series

$$
\begin{aligned}
& \left\{\begin{array}{c}
W(x, t) \\
V^{A}(x, t)
\end{array}\right\}=\sum_{m=1}^{n}\left\{\begin{array}{l}
w_{m}(t) \\
v_{m}^{A}(t)
\end{array}\right\} X_{m}(x) \\
& \left\{\begin{array}{c}
Q(x, t) \\
Q^{A}(x, t)
\end{array}\right\}=\sum_{m=1}^{n}\left\{\begin{array}{l}
q_{m}(t) \\
q_{m}^{A}(t)
\end{array}\right\} X_{m}(x) \quad A=1, \ldots, N
\end{aligned}
$$

For the hinged-hinged boundary conditions, the linear natural vibration modes are

$$
X_{m}(x)=\sin \left(\xi_{m}\right) x \quad \xi_{m}=\frac{m \pi}{L}
$$

Application of the Galerkin method leads to a system of $m \times(1+N)$ ordinary differential equations

$$
\mathbf{K y}+\mathbf{M} \ddot{\mathbf{y}}=\mathbf{q} \quad \mathbf{K}=\mathbf{K}_{0}+\mathbf{K}_{N L}(\mathbf{y})
$$

where

$$
\mathbf{y}=\mathbf{y}(t)=\left\{\begin{array}{lllllllll}
w_{1}(t) & w_{2}(t) & \cdots & v_{1}^{1}(t) & v_{2}^{1}(t) & \cdots & v_{1}^{2}(t) & v_{2}^{2}(t) & \cdots
\end{array}\right\}^{\mathrm{T}}
$$

The linear natural frequencies and mode shapes are sought for as solutions to the linearized eigenproblem

$$
\left|\mathbf{K}_{0}-\omega^{2} \mathbf{M}\right|=0
$$

In the case of free and forced nonlinear vibrations, equations (6.4)1can be converted into a system of the first order ordinary differential equations

$$
\ddot{\mathbf{y}}=\mathbf{M}^{-1}(\mathbf{q}-\mathbf{K y}) \Leftrightarrow\left\{\begin{array}{l}
\dot{\mathbf{y}}=\mathbf{v} \\
\dot{\mathbf{v}}=\mathbf{M}^{-1}(\mathbf{q}-\mathbf{K} \mathbf{y})
\end{array}\right.
$$

and solved by forward numerical integration. The calculations have been performed in Maple, using own procedure based on the Runge-Kutta-Fehlberg (RKF45) method.

\section{Calculational results}

The length of the beam is $L=1.0 \mathrm{~m}$, Young's modulus $E=205 \mathrm{GPa}$, mass density $\rho=$ $7850 \mathrm{~kg} / \mathrm{m}^{3}$. The other dimensionless parameters are as follows

$$
\begin{array}{llll}
\frac{b}{h}=5 & \frac{h}{l}=0.1 & \frac{l}{L}=0.1 & \frac{M_{1}}{\rho A l}=5.1 \\
\frac{M_{2}}{M_{1}}=0.5 & \sqrt{\frac{I_{1}}{M_{1} l^{2}}}=10 & \frac{I_{2}}{I_{1}}=0.5 &
\end{array}
$$


Let us introduce a dimensionless central macrodeflection, deflection fluctuation and load amplitude

$$
w=\frac{W_{\text {center }}}{h} \quad v=\frac{V_{\text {center }}}{h} \quad p=\frac{1}{h} \frac{q_{0} L^{4}}{\pi^{2} E J}
$$

In the forced vibrations analysis, the load frequency is $5 / 4$ times the lowest free undamped frequency of the beam

$$
\Omega=\frac{5}{4} \omega_{1}
$$

\subsection{Free undamped vibrations}

We restrict ourselves to considering only the first two $(m=2)$ terms of Fourier series (6.2) and two FSFs $(N=2)$ so that the model has $m(1+N)=6$ degrees of freedom.

In order to validate the results, a finite element method procedure for beam dynamics analysis has been written in Maple. The Rayleigh beam elements with Hermitian polynomials and consistent mass matrix have been applied.

The results of comparative analysis of calculations obtained for the finite element (40 elements) model and the tolerance model, using the approximate (trigonometric) and refined (finite element based) fluctuation shape functions (FSF), are shown in Table 1 (linear eigenfrequencies) and Fig. 5 (linear eigenvectors).

Table 1. Comparison of linear eigenfrequencies of the considered beam

\begin{tabular}{|c|c|c|c|c|c|}
\hline \multirow{2}{*}{ Mode } & \multirow{2}{*}{$\begin{array}{c}\text { Finite } \\
\text { element }\end{array}$} & \multicolumn{4}{|c|}{ Tolerance model } \\
\cline { 3 - 6 } & $\omega_{F E}[\mathrm{rad} / \mathrm{s}]$ & $\omega_{T A}[\mathrm{rad} / \mathrm{s}]$ & $\Delta[\%]$ & $\omega_{T A}[\mathrm{rad} / \mathrm{s}]$ & $\Delta[\%]$ \\
\hline \hline $1 . w_{1}$ & 15.861 & 15.876 & 0.094 & 15.872 & 0.071 \\
\hline $2 . w_{2}$ & 32.884 & 33.050 & 0.505 & 33.041 & 0.477 \\
\hline $3 . v_{2}^{2}$ & 223.491 & 239.454 & 7.143 & 212.200 & 5.052 \\
\hline $4 . v_{1}^{2}$ & 224.244 & 246.109 & 9.751 & 224.088 & 0.069 \\
\hline $5 . v_{2}^{1}$ & 14855.007 & 15129.460 & 1.848 & 15034.591 & 1.209 \\
\hline $6 . v_{1}^{1}$ & 14989.493 & 15129.460 & 0.934 & 15034.591 & 0.301 \\
\hline
\end{tabular}

Figure 6 presents the total central deflection versus the quotient of nonlinear frequency to linear frequency of free macro- and micro-vibrations (backbone curves) corresponding to the first macro-mode and symmetric cell vibrations $(m=1, N=1)$. Parameters (7.1) are kept constant except the dimensionless microstructure parameter $\lambda=l / L$ that is equal to $1 / 10,1 / 12$ or $1 / 15$.

Studying the forced vibrations, the first 200 load periods are considered. The bifurcation diagram of the total dimensionless deflection $w$ at $x=0.5 L$ versus the dimensionless load amplitude is shown in Fig. 7. Figure 8 presents a close-up of the bifurcation diagram of deflection together with the diagram of its velocity $d w / d t$.

\section{Discussion of results}

From the results of linear free vibrations analysis, it can be seen that the finite element based fluctuation shape functions indicate a better performance than the first-term trigonometric ones, although the significant differences appear only for the antisymmetric mode shapes of the periodicity cell. This applies both to the frequencies (Table 1) and the mode shapes (Fig. 5). It should be noted that approximation of the lowest order possible is applied in the numerical 
(a)
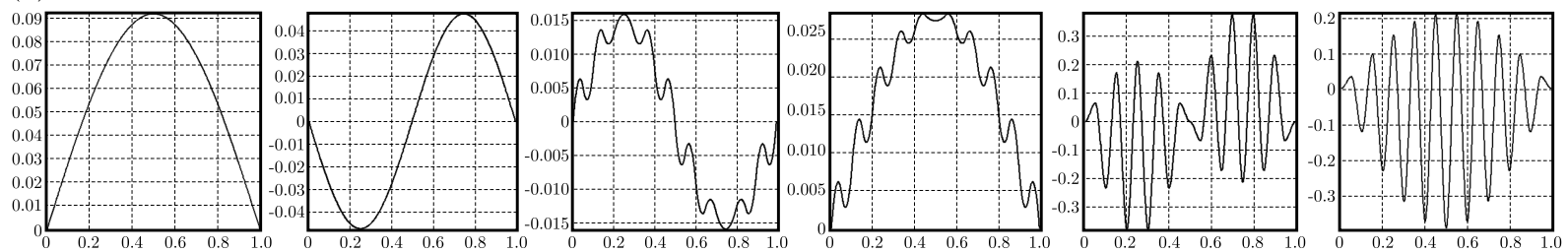

(b)
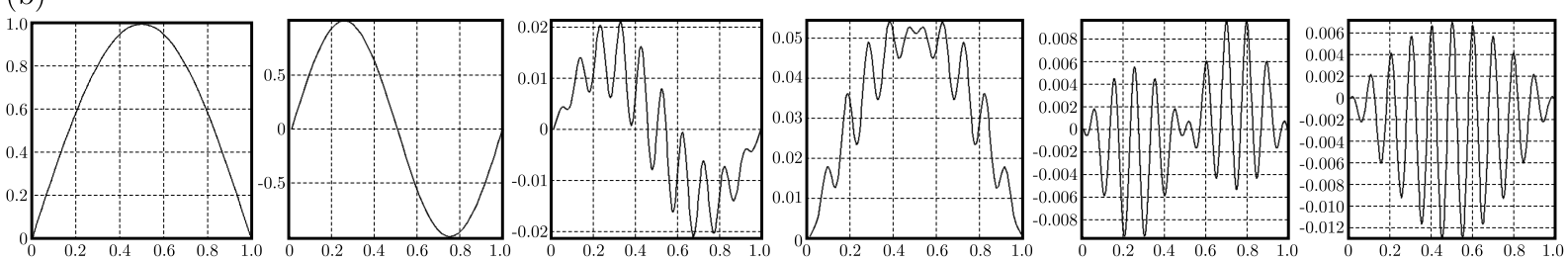

(c)
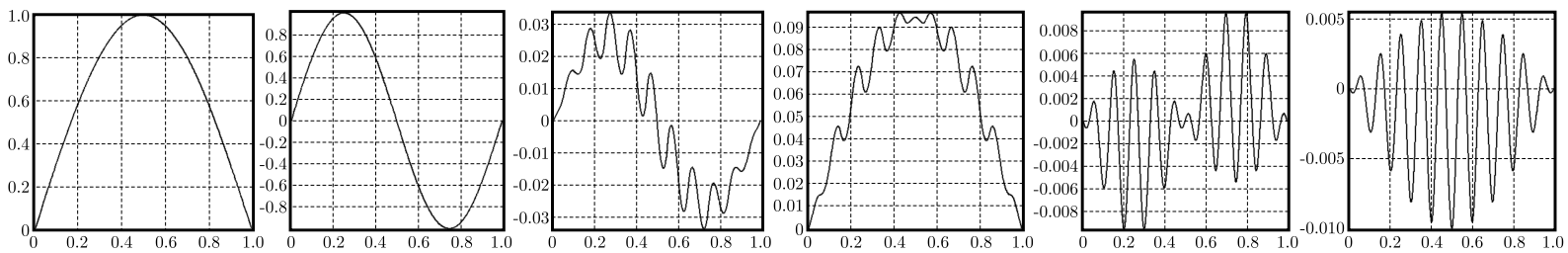

Fig. 5. Comparison of linear eigenforms of the considered beam: (a) finite element model, (b) tolerance model with trigonometric FSFs, (c) tolerance model with FE-based FSFs

(a)

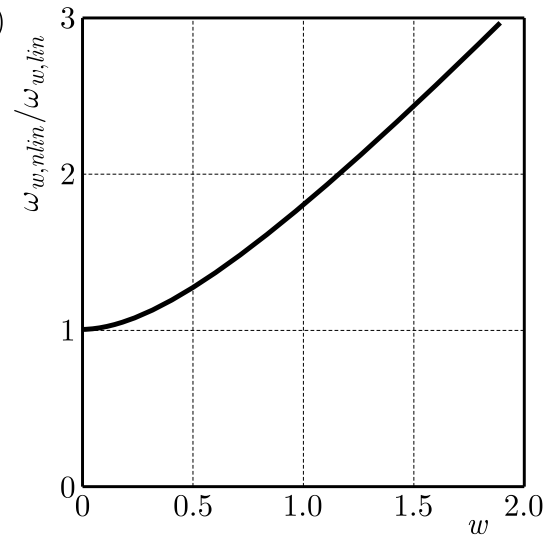

(b)

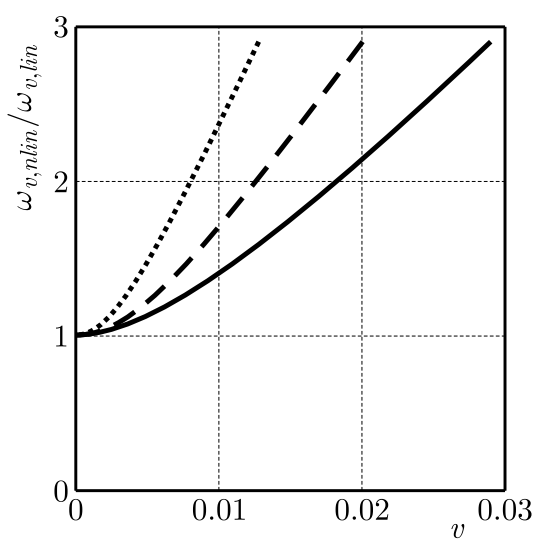

Fig. 6. Backbone curves for dimensionless macrodeflection (a) and fluctuation (b) at $x=0.5 L$ for $\lambda=1 / 10$ (solid line), $\lambda=1 / 12$ (dashed line), $\lambda=1 / 15$ (dotted line)

analysis on the macro- and microlevel. As it is mentioned above, these results can be improved through a more accurate analysis of the cell problem.

When it comes to nonlinear free vibrations the backbone curves presented in Fig. 6 indicate that the lower order (macro-) nonlinear vibrations frequency is practically not affected by the periodicity cell length, while the higher order frequency is much more sensitive to its variation. It is caused by the fact that in the geometrically nonlinear formulation the strain terms that include the displacement fluctuations are dependent on the microstructure parameter $l$, what does not take place in the linear model.

Studying the results of the forced vibrations case (Figs. 7 and 8), the most characteristic feature is that the bifurcation diagram is very scattered, although there are many narrow periodic windows. This is due to the fact that free vibrations are not damped, so they do not fade with time. For the values of $p$ higher than $\sim 3$, it can be seen that the envelope of the diagram suddenly loses its regularity and becomes jagged, which may indicate irregular vibrations. This requires a more detailed analysis. 


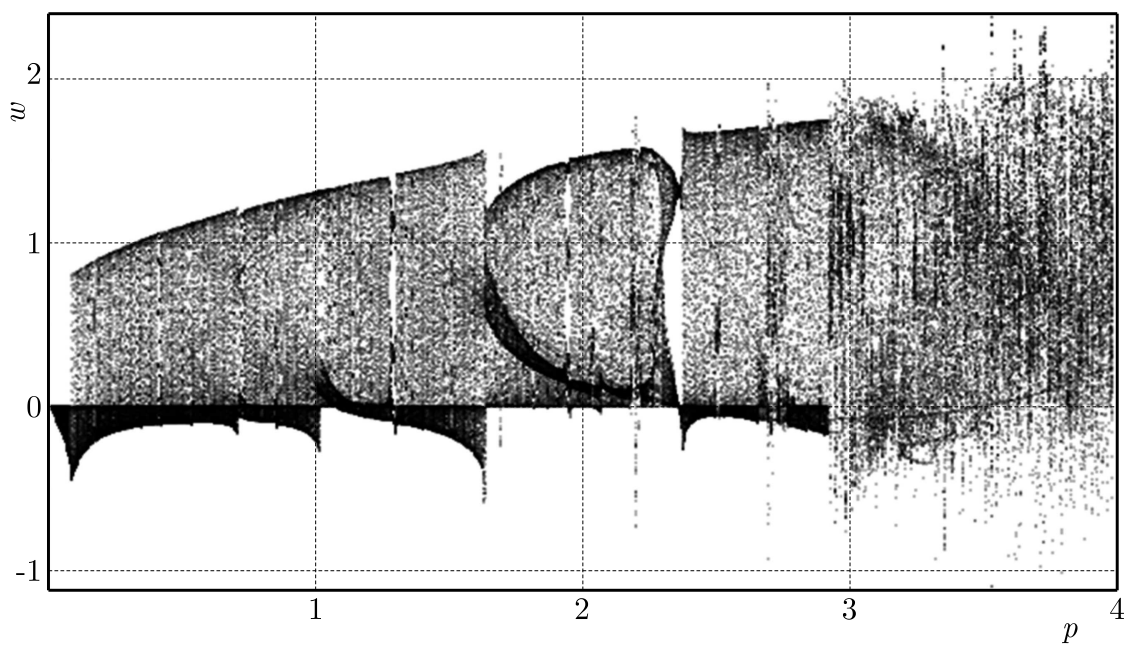

Fig. 7. The bifurcation diagram of the deflection $w$ vs. the load amplitude $p$
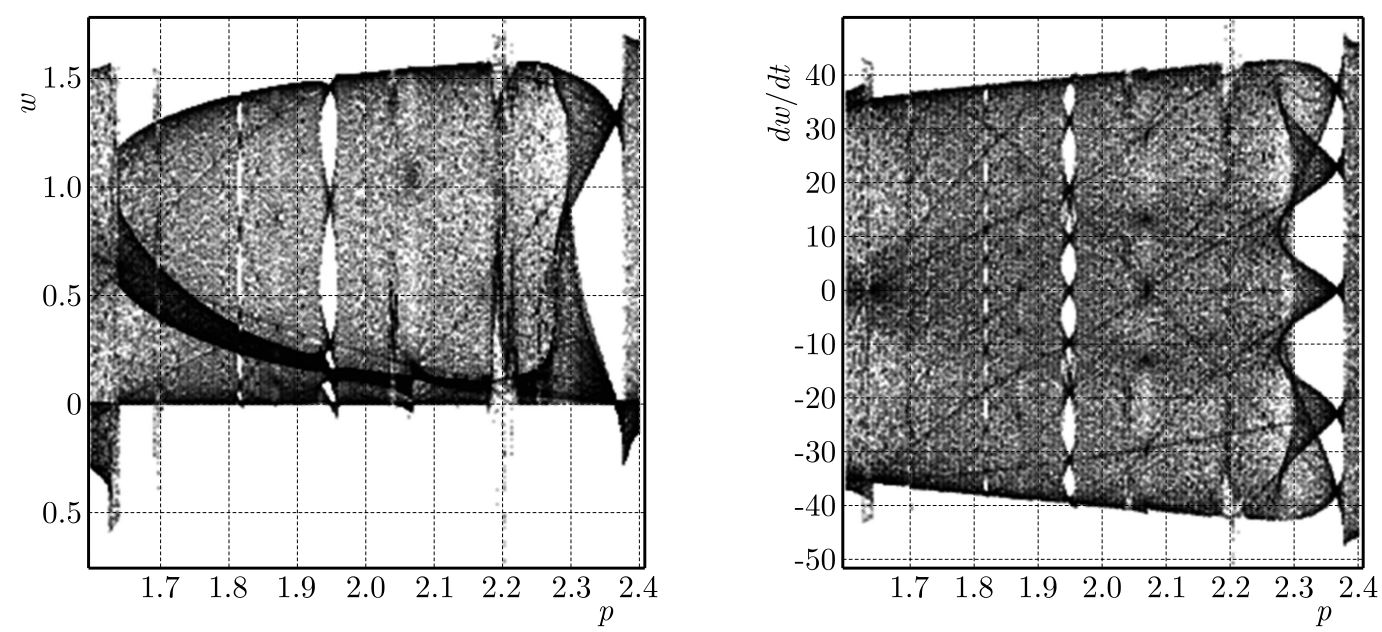

Fig. 8. A blow-up of the bifurcation diagram: the deflection $w$ and the velocity $d w / d t$ vs. the load amplitude $p$

\section{Closing remarks}

In this contribution, the tolerance model is shown, which describes geometrically nonlinear vibrations of a periodically inhomogeneous beam. The model is developed by applying the tolerance averaging method directly to the 1D beam theory equations. Hence, the fundamental equations with highly oscillating, periodic, non-continuous functional coefficients are replaced by the equations with constant coefficients. It should be stressed that the aim is to develop a low degree of freedom model that would be able to provide results that are not available for the first order asymptotic models. Some applications of the proposed model including free and forced undamped vibrations are presented. A comparison of linear eigenfrequencies and mode shapes with a finite element model is shown.

The following general remarks can be formulated.

1. It can be observed that the proposed toleranc emodel makes it possible to investigate the effect of the microstructure size on dynamic problems of periodic beams under consideration, e.g. the "higher order" vibrations related to the beam microstructure.

2. The governing equations of the tolerance model have a physical sense for the unknowns $W, U, V^{A}, A=1, \ldots, N, T^{K}, K=1, \ldots, M$, being slowly-varying functions. 
3. The asymptotic model of periodic beams makes it possible to investigate only lower order (fundamental) vibrations.

The issues anticipated to be addressed in the future work are:

- taking into account the structural and material heterogeneity of the beam and the viscoelastic subsoil,

- more detailed analysis of the solutions to the cell problem,

- detailed analysis of the properties distribution in a periodicity cell,

- analysis of the initial shortening/elongation of the beam axis.

These problems will be investigated in forthcoming papers.

\section{Acknowledgement}

The authors are grateful for the support provided by the National Science Centre, Poland (Grant No. 2014/15/B/ST8/03155).

\section{References}

1. Awrejcewicz J., Krysko A.V., Mrozowski J., Saltykova O.A., Zhigalov M.V., 2011, Analysis of regular and chaotic dynamics of the Euler-Bernoulli beams using finite difference and finite element methods, Acta Mechanica Sinica, 27, 36-43

2. Bakhvalov N.S., Panasenko G.P., 1984, Averaging of Processes in Periodic Media (in Russian), Nauka, Moskwa

3. BAnakh L., Kempner M., 2010, Vibrations of mechanical systems with regular structure, [In:] Foundations of Engineering Mechanics, Springer, Berlin

4. BARON E., 2006, On modelling of periodic plates having the inhomogeneity period of an order of the plate thickness, Journal of Theoretical and Applied Mechanics, 44, 3-18

5. Bensoussan A., Lions J.L., Papanicolaou G., 1978, Asymptotic Analysis for Periodic Structures, North-Holland, Amsterdam

6. Chen T., 2013, Investigations on flexural wave propagation of a periodic beam using multireflection method, Archive of Applied Mechanics, 83, 315-329

7. Chen T., Wang L., 2013, Suppression of bending waves in a periodic beam with Timoshenko beam theory, Acta Mechanica Solida Sinica, 26, 177-188

8. He W.M., Chen W.Q., Qiao H., 2013, Frequency estimate and adjustment of composite beams with small periodicity, Composites: Part B, 45, 742-747

9. Hryniewicz Z., Kozio€ P., 2013, Wavelet-based solution for vibrations of a beam on a nonlinear viscoelastic foundation due to moving load, Journal of Theoretical and Applied Mechanics, 51, $215-224$

10. JĘDRYSIAK J., 1999, Dynamics of thin periodic plates resting on a periodically inhomogeneous Winkler foundation, Archive of Applied Mechanics, 69, 345-356

11. JĘDRYSIAK J., 2013, Modelling of dynamic behaviour of microstructured thin functionally graded plates, Thin Walled Structures, 71, 102-107

12. JęDRYSiAK J., Woźniak C., 2006, On the propagation of elastic waves in a multiperiodically reinforced medium, Meccanica, 41, 553-569

13. Jikov V.V., Kozlov S.M., OleiniK O.A., 1994, Homogenization of Differential Operators and Integral Functionals, Springer Verlag, Berlin-Heidelberg-New York

14. Kolpakov A.G., 1991, Calculation of the characteristics of thin elastic rods with a periodic structure, Journal of Applied Mathematics and Mechanics, 55, 358-365

15. Kolpakov A.G., 1995, The asymptotic theory of thermoelastic beams, Journal of Applied Mechanics and Technical Physics, 36, 756-763 
16. Kolpakov A.G., 1998, Application of homogenization method to justification of 1-D model for beam of periodic structure having initial stresses, International Journal of Solids and Structures, 35, 2847-2859

17. Kolpakov A.G., 1999, The governing equations of a thin elastic stressed beam with a periodic structure, Journal of Applied Mathematics and Mechanics, 63, 495-504

18. Krysko A.V., Zhigalov M.V., Saltykova O.A., 2008, Control of complex nonlinear vibrations of sandwich beams, Russian Aeronautics, 51, 238-243

19. Lewiński T., Telega J..J., 2000, Plates, laminates and shells, World Scientific Publishing Company, Singapore

20. Magnucki K., Jasion P., Krus M., Kuligowski P., Wittenbeck L., 2013, Strength and buckling of sandwich beams with corrugated core, Journal of Theoretical and Applied Mechanics, $\mathbf{5 1}, 15-24$

21. Mazur-ŚniAdy K., 1993, Macro-dynamics of micro-periodic elastic beams, Journal of Theoretical and Applied Mechanics, 31, 781-793

22. Mazur-Śniady K., ŚniAdy P., 2001, Dynamic response of a micro-periodic beam under moving load - deterministic and stochastic approach, Journal of Theoretical and Applied Mechanics, 39, 323-338

23. Mazur-Śniady K., Woźniak C., Wierzbicki E., 2004, On the modelling of dynamicproblems for plates with a periodic structure, Archive of Applied Mechanics, 74, 179-190

24. Olhoff N., Niu B., Cheng G., 2012, Optimum design of band-gap beam structures, International Journal of Solids and Structures, 49, 3158-3169

25. Sanchez-Palencia E., 1980, Non-Homogeneous Media and Vibration Theory, Lecture Notes in Physics 127, Springer-Verlag, Berlin

26. Sedighi H.M., Reza A., Zare J., 2013, The effect of quintic nonlinearity on the investigation of transversely vibrating buckled Euler-Bernoulli beams, Journal of Theoretical and Applied Mechanics, 51, 959-968

27. Syerko E., Diskovsky A.A., Andrianov I.V., Comas-Cardona S., Binetruy C., 2013, Corrugated beams mechanical behavior modelling by the homogenization method, International Journal of Solids and Structures, 50, 928-936

28. Sylvia J.E., Hull A.J., 2013, A dynamic model of a reinforced thin plate with ribs of finite width, International Journal of Acoustics and Vibration, 18, 86-90

29. TомсzYK B., 2007, A non-asymptotic model for the stability analysis of thin biperiodic cylindrical shells, Thin Walled Structures, 45, 941-944

30. WANG Y.Z., Li F.M., 2014, Nonlinear primary resonance of nano beam with axial initial load by nonlocal continuum theory, International Journal of Non-Linear Mechanics, 61, 74-79

31. Wirowski A., 2012, Self-vibration of thin plate with non-linear functionally graded material, Archives of Mechanics, 64, 603-615

32. Woźniak C. ET AL. (Eds.), 2010, Mathematical Modelling and Analysis in Continuum Mechanics of Microstructured Media, Silesian University of Technology Press, Gliwice, Poland

33. Woźniak C., Michalak B., Jędrysiak J. (EDs.), 2008, Thermomechanics of Microheterogeneous Solids and Structures. Tolerance Averaging Approach, Lodz Technical University Press, Łódź, Poland

34. Woźniak C., WierzBicki E., 2000, Averaging Techniques in Thermomechanics of Composite Solids, Czestochowa University of Technology Press, Częstochowa, Poland

35. Yu D., Wena J., Shen H., Xiao Y., Wen X., 2012, Propagation of flexural wave in periodic beam on elastic foundations, Physics Letters A, 376, 626-630 